\title{
Approximate Operational Calculus in Chemical Engineering
}

STUART W. CHURCHILL

University of Michigan, Ann Arbor, Michigan

Approximations are suggested to extend the usefulness of operational calculus in solving boundary-value problems of interest to the chemical engineer. General approximations are outlined and specific ones illustrated. The use of computing machines with operational calculus is also considered.

Operational calculus has been found useful in many characteristic problems in chemical engineering. The applications considered by Marshall and Pigford (6) and those noted in recent annual reviews ( 8 to 11) include diffusion, conduction, convection, distillation, kinetics, and process control.

Unfortunately, present operational techniques are not always adequate for the very problems for which, in principle, operational calculus holds the most promise. These limitations are aggravated by the notorious difficulty encountered by the mathematician in establishing proofs in operational calculus for procedures that he suspects to be valid.

Approximation can be used to surmount some of the limitations in operational theory. In addition to permitting solution of complex and otherwise intractable problems, approximation may indicate useful simplifications that are not apparent from an exact solution. The use of approximation has been rather slighted in the literature of operational calculus, presumably because of the taint imposed by lack of rigor. Although numerous specific examples having a physical or mathematical basis have been reported, only McLachlan (7) and Doetsch (3) have attempted to generalize the use of approximation, and then only for the limiting conditions of long and short times in transient problems.

Since the purpose of this paper is to acquaint the chemical engineer with the possibilities and advantages of a mathematical technique, rather than to develop new mathematics, all proofs and most details will be omitted. Approximations will generally be justified on physical grounds. The engineer is seldom disturbed by his inability to establish rigorously the validity or uniqueness of a solution if it satisfies physical tests.

Consideration will be limited to the Laplace transformation, which is the most extensively used form of operational calculus. The use of approximation with other transforms is also advantageous, and equivalent procedures are readily developed.

\section{THE LAPLACE TRANSFORMATION}

If a function $F^{\prime}(t)$ is multiplied by $e^{-s t}$ and integrated with respect to $t$ from zero to infinity, a new function, $f(s)$, is obtained. This operation is called the Laplace transformation of $F(t)$, and $f(s)$ is called the Laplace transform; i.e.,

$\mathscr{E}\{F(t)\}=\int_{0}^{\infty} e^{-s t} F(t) d t=f(s)$
The inverse operation is designated

$$
F(t)=\mathfrak{£}^{-1}\{f(s)\}
$$

Operational calculus was developed empirically by Heaviside as a set of rules of procedure. Subsequently an extensive theory was developed, and the mathematical foundations, limitations, and formal applications are well treated in modern texts $(2,7,14)$. Although transforms have been derived and tabulated for many functions and mathematical operations (4), problems of interest more often than not yield functions not to be found in such tables.

Letting the transform variable, $s$, be a complex variable permits expression of the inverse transformation in terms of the complex inversion integral.

$$
F(t)=\frac{1}{2 \pi i} \lim _{\beta \rightarrow \infty} \int_{\gamma-i \beta}^{\gamma+i \beta} e^{s t} f(s) d s
$$

where

$s=x+i y$

$\gamma=$ a sufficiently large, fixed value of $x$.

The theory of residues and line integrals can be used to evaluate the foregoing integral. In many cases the problem is reduced to one of finding the singularities in $f(s)$.

The complex inversion integral can be written as a real integral,

$$
\begin{array}{r}
F(t)=\frac{e^{\gamma t}}{\pi} \int_{0}^{\infty}[u(\gamma, y) \cos y t \\
-v(\gamma, y) \sin y t] d y
\end{array}
$$

where $u=$ real part of $f(s)$, and $v=$ imaginary part of $f(s)$, but in this form the integration is generally too difficult to be performed analytically.

The most important operational property of the Laplace transformation arises from the derivative

$$
\mathfrak{E}\left\{F^{\prime}(t)\right\}=s f(s)-F(+0)
$$

Differentiation of $F(t)$ thus corresponds to multiplication of the transform $f(s)$ by $s$ and subtraction of $F(+0)$. Equation (5) in turn leads to an expression for the transform of the $n$th derivative:

$$
\begin{aligned}
& £\left\{F^{n}(t)\right\}=s^{n} f(s)-s^{n-1} F(+0) \\
& -s^{n-2} F^{\prime}(+0)-\cdots-F^{n-1}(+0)
\end{aligned}
$$

Linear ordinary differential equations with constant coefficients are thus transformed into algebraic equations, solution of which followed by inversion then yields a solution to the differential equation. Other operational properties permit simplification and solution of a few more complex types of differential equations.

The transformation of simple partial differential equations yields ordinary differential equations, which may be easier to solve. The solution of the ordinary differential equation involving a transformed function may possibly be solvable by a second transformation.

Multiplication of the transforms of two functions corresponds to a particular integration

$$
\begin{aligned}
& \mathfrak{E}^{-1}\left\{f_{1}(s) \cdot f_{2}(s)\right\} \\
& =\int_{0}^{t} F_{2}(t-\lambda) F_{1}(\lambda) d \lambda
\end{aligned}
$$

This property permits solution of certain integral equations as well as additional differential equations.

The operation

$$
£\{F(t-m)\}=e^{-m s} f(s)
$$

where

$$
F(t)=0 \text { for } t<0
$$

permits solution of certain finite-difference equations and finite-differencedifferential equations such as those encountered in equilibrium-stage operations.

From these examples, several limitations on the usefulness of the operational method are apparent or implied:

1. The functions encountered in the equations and boundary conditions must be transformable.

2. The transformed equations and boundary conditions must be solvable.

3. It must be possible to invert the transformed solution.

The mathematical restrictions on the transformation are not very serious and are satisfied by most functions encountered in engineering problems. On the other hand, the class of equations which are simplified by transformation is rather limited. With such equations the relative 
advantage of the operational method over other classical methods depends largely on the nature of the boundary conditions. In particular, if the values of the dependent variable to be transformed and its derivatives at $t=+0$ are known, the operational method is at least worth looking into. The simplification gained by transformation is often negated by difficulties in inverting the transformed solution.

\section{APPROXIMATE INVERSION}

The inversion process may be simplified or expedited by the following methods, one or more of which are applicable in most problems.

1. The transformed function may be expanded in series. The entire series may then be inverted or appropriate terms may be discarded before inversion. Frequently several different expansions are feasible, yielding solutions or indicating approximations of different utility. The use of series expansion is illustrated in Problems I, II, and III, which follow.

2. A complicated function may be represented empirically by some arbitrary function which is more readily inverted, as indicated in Problem III. This method of approximation can seldom be justified mathematically but is worth trying if the solution can be tested. (Similarly, boundary conditions and generating functions may be approximated by other functions more susceptible to transformation, as illustrated in Problem IV.)

3. The inversion may be carried out only for particular or limiting values of a parameter, as indicated in Problems I and III, or of a nontransformed independent variable, as indicated in Problem II. This procedure may be expressed mathematically as

$$
\begin{aligned}
F\left(x_{0}, t\right) & =[F(x, t)]_{x=x_{0}} \\
& =\left\{\mathfrak{E}^{-1}[f(x, s)]\right\}_{x=x_{0}} \\
& =\mathfrak{E}^{-1}\left[f\left(x_{0}, s\right)\right]
\end{aligned}
$$

4. Approximate inversions for large and small values of $t$ can sometimes be obtained by letting $s$ assume small and large values, respectively; i.e.,

and

$$
\underset{t \rightarrow \infty}{F(t)} \cong \mathfrak{f}^{-1}\{\underset{s \rightarrow 0}{f(s)\}}
$$

$$
\underset{t \rightarrow 0}{(F t)} \cong \mathfrak{£}^{-1}\{f(s)\}
$$

One or both of these approximations are illustrated in Problems I, II, and III. Equation (10) is not valid if any of the singularities in $f(s)$ occur in the half of the complex plane where the real value of $s$ is positive. Thus formal use of this approximation may lead to error; however, it is usually easier to detect an erroneous solution than to find the singularities.

5. The real form of the inversion integral, Equation (4), can be evaluated graphically, numerically, or by quadrature, as suggested in Problem III.

6. Approximate values may be used for the singularities when residue theory is used to carry out the inversion. This approximation is not used in any of the illustrative problems.

\section{ILLUSTRATIVE PROBLEMS}

Four problems were chosen to illustrate the use of approximation with the Laplace transformation. The equations in the problems are streamlined by the implicit inclusion of physical factors and constants in generalized variables.

Although the greatest utility of approximation is in complex problems that cannot be solved rigorously, relatively simple problems were deemed best for illustration. In several cases the nature and validity of the approximations are apparent by direct comparison with a rigorous solution. The techniques used in these simple problems are of course directly applicable in more complex ones.

\section{Problem I}

Jeffreys (5) has indicated the use of approximation in the solution of the set of simultaneous rate equations describing a radioactive decay series. $\mathrm{He}$ also discusses the physical interpretation of the approximate results in some detail. The general problem is represented by the equations

$$
\frac{d C_{1}}{d t}=-k_{1} C_{1} \text { with } C_{1}=C_{0} \text { at } t=0
$$

$$
\begin{aligned}
& \frac{d C_{2}}{d t}=-k_{2} C_{2}+k_{1} C_{1} \\
& \frac{d C_{3}}{d t}=-k_{3} C_{3}+k_{2} C_{2}
\end{aligned}
$$

etc., and finally

$$
\frac{d C_{n}}{d t}=k_{n-1} C_{n-1}
$$

for the end product, which does not decompose.

Transformation yields the corresponding equations

$c_{1}=\frac{C_{0}}{s+k_{1}}$

$c_{2}=\frac{k_{1} C_{0}}{\left(s+k_{2}\right)\left(s+k_{1}\right)}$

$$
c_{3}=\frac{k_{2} k_{1} C_{0}}{\left(s+k_{3}\right)\left(s+k_{2}\right)\left(s+k_{1}\right)}
$$

and

$c_{n}=\frac{k_{n-1} k_{n-2} \cdots k_{1} C_{0}}{s\left(s+k_{n-1}\right)\left(s+k_{n-2}\right) \cdots\left(s+k_{1}\right)}$
The corresponding exact solutions are

$$
\begin{gathered}
C_{\mathbf{1}}=C_{0} e^{-k_{1} t} \\
C_{2}=k_{1} C_{0}\left[\frac{e^{-k_{1} t}}{k_{2}-k_{1}}+\frac{e^{-k_{2} t}}{k_{1}-k_{2}}\right] \\
C_{3}=k_{2} k_{1} C_{0}\left[\frac{e^{-k_{1} t}}{\left(k_{3}-k_{1}\right)\left(k_{2}-k_{1}\right)}\right. \\
+\frac{e^{-k_{2} t}}{\left(k_{3}-k_{2}\right)\left(k_{1}-k_{2}\right)} \\
\left.+\frac{e^{-k_{3} t}}{\left(k_{2}-k_{3}\right)\left(k_{1}-k_{3}\right)}\right]
\end{gathered}
$$

and

$$
\begin{array}{r}
C_{n}=C_{0}-k_{n-1} k_{n-2} \cdots k_{1} C_{0} \\
\cdot\left[\frac{e^{-k_{1} t}}{k_{1}\left(k_{n-1}-k_{1}\right)\left(k_{n-2}-k_{1}\right) \cdots\left(k_{2}-k_{1}\right)}\right. \\
+\cdots]
\end{array}
$$

In the following approximations $s$ will be treated as a real variable.

\section{Short-time Approximation}

For very short times $|s|$ will be very large with respect to all the rate constants. Then

$$
\begin{aligned}
c_{1} & \rightarrow \frac{C_{0}}{s} \\
c_{2} & \rightarrow \frac{k_{1} C_{0}}{s^{2}} \\
c_{3} & \rightarrow \frac{k_{2} k_{1} C_{0}}{s^{3}} \\
c_{n} & \rightarrow \frac{k_{n-1} k_{n-2} \cdots k_{1} C_{0}}{s^{n}}
\end{aligned}
$$

and

$$
\begin{aligned}
& C_{1} \rightarrow C_{0} \\
& C_{2} \rightarrow k_{1} C_{0} t \\
& C_{3} \rightarrow \frac{k_{2} k_{1} C_{0} t^{2}}{2 !} \\
& C_{n} \rightarrow \frac{k_{n-1} k_{n-2} \cdots k_{1} C_{0} t^{n-1}}{(n-1) !}
\end{aligned}
$$

\section{Moderate-time Approximation}

Expanding the transforms in series by division yields a useful form for moderately short times corresponding to $|s|$ greater than any $k$. Thus

$$
c_{1}=\frac{C_{0}}{s}\left[1-\left(\frac{k_{1}}{s}\right)+\left(\frac{k_{1}}{s}\right)^{2}+\cdots\right]
$$

$$
\begin{aligned}
c_{2} & =\frac{k_{1} C_{0}}{s^{2}}\left[1-\frac{\left(k_{1}+k_{2}\right)}{s}\right. \\
& \left.+\frac{\left(k_{1}{ }^{2}+k_{1} k_{2}+k_{2}{ }^{2}\right)}{s^{2}}+\cdots\right]
\end{aligned}
$$




$$
\begin{aligned}
c_{3} & =\frac{k_{2} k_{1} C_{0}}{s^{3}} \\
\cdot & {\left[1-\frac{\left(k_{1}+k_{2}+k_{3}\right)}{s}+\cdots\right] } \\
c_{n} & =\frac{k_{n-1} k_{n-2} \cdots k_{1} C_{0}}{s^{n}} \\
& {\left[1-\frac{k_{1}+k_{2} \cdots+k_{n-1}}{s}+\cdots\right] }
\end{aligned}
$$

and

$$
\begin{aligned}
C_{1}=C_{0}\left[1-k_{1} t+\frac{\left(k_{1} t\right)^{2}}{2 !}\right. & \left.-\frac{\left(k_{1} t\right)^{3}}{3 !}+\cdots\right] \\
C_{2}= & k_{1} C_{0} t\left[1-\frac{\left(k_{1}+k_{2}\right) t}{2 !}\right. \\
+ & \left.\frac{\left(k_{1}^{2}+k_{2} k_{1}+k_{2}{ }^{2}\right)}{3 !}-\cdots\right]
\end{aligned}
$$$$
C_{3}=\frac{k_{2} k_{1} C_{0} t^{2}}{2 !}
$$$$
\left[1-\frac{\left(k_{1}+k_{2}+k_{3}\right) t}{3 !}+\cdots\right]
$$$$
C_{n}=\frac{k_{n-1} k_{n-2} \cdots k_{1} C_{0} t^{n-1}}{(n-1) !}
$$$$
\left[1-\frac{\left(k_{1}+k_{2}+\cdots k_{n-1}\right) t}{n !}+\cdots\right]
$$

The first terms of the series solutions are the very short-time solutions. The series themselves are obviously those obtained from series expansion of the exact solutions. Thus in this problem a shorttime approximation of any desired accuracy can be obtained by letting $|s| \rightarrow \infty$ and dropping higher order terms.

\section{Long-time Approximation}

For long times $s$ will be small with respect to some of the rate constants. If it is assumed that all the rate constants except $k_{2}$ are much larger than $|s|$

$$
\begin{gathered}
c_{1} \rightarrow \frac{C_{0}}{k_{1}} \text { and } C_{1} \rightarrow 0 \\
c_{2} \rightarrow \frac{C_{0}}{s+k_{2}}, \\
\quad \text { and } C_{2} \rightarrow C_{0} e^{-k_{2} t} \\
c_{3} \rightarrow \frac{k_{2} C_{0}}{k_{3}\left(s+k_{2}\right)}, \\
\text { and } C_{3} \rightarrow \frac{k_{2} C_{0}}{k_{3}} e^{-k_{2} t}
\end{gathered}
$$

$c_{n} \rightarrow \frac{k_{2} C_{0}}{s\left(s+k_{2}\right)}$,

$$
\text { and } C_{n} \rightarrow C_{0}\left[1-e^{-k_{2} t}\right]
$$

Equation (41) does not follow directly from Equation (40) but is apparent from Equation (12) or (20). These approximate solutions can, of course, be obtained from the exact solution. Thus the formal procedure used to obtain the approximations is substantiated in this case even though it is not in general rigorous.

\section{Problem II}

The representation of a transformed solution by an asymptotic series to expedite inversion or to yield a more satisfactory solution upon inversion has been used widely. The following example from Carslaw and Jaeger (1) also illustrates inversion at a single value of the untransformed variable.

The transformed solution for the temperature in a long cylindrical rod, initially at zero temperature but maintained at unit temperature at the surface after time zero, can be written in generalized terms as

$$
u(r, s)=\frac{I_{0}(\sqrt{s r})}{s I_{0}(\sqrt{s})}
$$

The details of the derivation of Equation (48) are given in reference 1 . The exact solution is

$T(r, t)=1-2 \sum_{n=1}^{\infty} \frac{J_{0}\left(\alpha_{n} r\right)}{\alpha_{n} J_{1}\left(\alpha_{n}\right)} e^{-\alpha_{n}^{2} t}$

$$
u(s)=\frac{1}{s\left[1-\sqrt{s}\left(\frac{1+\left(\frac{1-L \sqrt{s}}{1+L \sqrt{s}}\right) e^{2 \sqrt{s}(L-1)}}{1-\left(\frac{1-L \sqrt{s}}{1+L \sqrt{s}}\right) e^{2 \sqrt{s}(L-1)}}\right)+\phi s\right]}
$$

where $\alpha_{n}$ are the roots of $J_{0}\left(\alpha_{n}\right)=0$.

\section{Short-time Approximation}

Equation (49) is inconvenient for $t<0.02$ because the series converges very slowly. For short times and large $s$ only the positive exponentials of the asymptotic series

$$
\begin{aligned}
I_{0}(x) & =\frac{e^{x}}{\sqrt{2 \pi x}}\left[1+\frac{1}{8 x}\right. \\
+ & \left.\frac{9}{128 x^{2}}+\cdots\right]+\frac{e^{-x+1 / 2 \pi i}}{\sqrt{2 \pi x}} \\
& {\left[1-\frac{1}{8 x}+\frac{9}{128 x^{2}}-\cdots\right] }
\end{aligned}
$$

are significant. Introducing these terms into Equation (48) and simplifying gives

$$
\begin{gathered}
u(r, s) \cong \frac{e^{-\sqrt{s}(1-r)}}{s \sqrt{r}}\left[1+\frac{1-r}{8 r \sqrt{s}}\right. \\
\left.+\frac{9-2 r-7 r^{2}}{128 s r^{2}}+\cdots\right]
\end{gathered}
$$

Although all the terms of Equation (51) could be inverted, only the first term need be considered for short times if $r$ is not too small:

$$
T(r, t) \cong \frac{1}{\sqrt{r}} \operatorname{erfc}\left(\frac{1-r}{2 \sqrt{t}}\right)
$$

\section{Short-time Approximation for Center}

Putting $r=0$ in Equation (48) and then introducing the positive exponentials of the asymptotic series only in the denominator gives

$$
\begin{aligned}
u(0, s) \cong \frac{\sqrt{2 \pi}}{s^{3 / 4}} e^{-\sqrt{s}} & {\left[1-\frac{1}{8 \sqrt{s}}-\cdots\right] }
\end{aligned}
$$

For short times, only the first term needs to be inverted and

$$
T(0, t) \cong \frac{e^{-1 / 8 t}}{\sqrt{\pi t}} K_{1 / 4}\left(\frac{1}{8 t}\right)
$$

\section{Problem III}

Sleicher and Churchill (12) used a number of techniques to invert the following expression for the transient temperature of a sphere in a dispersion of spheres exposed to a radiant flux

The details of the derivation of Equation (55) and the physical significance of the variables are given in the foregoing reference.

\section{Short-time Approximation}

For large $s$ and $L>1$

$$
u(s) \rightarrow \frac{1}{s(1+\sqrt{s}+\phi s)}
$$

Equation (56) is also obtained by letting $L \rightarrow \infty$, which corresponds to reduction of the problem to one of a single sphere in an infinite medium.

\section{Integral Inversion}

The inverse transform of Equation (56) can be written in terms of the real integral

$$
T=1-\frac{2}{\pi \sqrt{\phi}}
$$




$$
\cdot \int_{0}^{\infty} \frac{e^{-(t / \phi) y^{2}} d y}{1-\left(2-\frac{1}{\phi}\right) y^{2}+y^{4}}
$$

The integral can be evaluated graphically for a series of values of $t$ and $\phi$. Accurate evaluation at small values of $t / \phi$ is difficult owing to a sharp peak in the integrand at $y=\sqrt{1-(1 / 2 \phi)}$. This difficulty can be minimized by rearranging Equation (57) as

$$
\begin{gathered}
T=1-e^{-t / \phi}\left[1-\frac{2}{\pi \sqrt{\phi}}\right. \\
\left.\cdot \int_{0}^{\infty} \frac{1-e^{-(t / \phi)\left(y^{2}-1\right)}}{1-\left(2-\frac{1}{\phi}\right) y^{2}+y^{4}} d y\right]
\end{gathered}
$$

or by taking a mean value of the exponential at the peak; i.e.,

$$
T \cong 1-\exp \left\{-\frac{t}{\phi}\left(1-\frac{1}{2 \phi}\right)\right\}
$$

As an example of the difficulty that can arise from an improper approximation, it will be noted that neglecting $1 / \phi$ with respect to 2 in the denominator of the integrand produces a zero in the denominator at $y=1$ and thus makes the integrand infinite.

\section{Series Inversion}

Formally carrying out the division indicated in Equation (56) yields

$$
\begin{aligned}
u=\frac{1}{s^{2} \phi} & {\left[1-\frac{1}{\phi \sqrt{s}}\right.} \\
& \left.-\frac{(\phi-1)}{\phi^{2} s}+\cdots\right]
\end{aligned}
$$

and inversion gives

$$
\begin{aligned}
T=\frac{t}{\phi} & {\left[1-\frac{4}{3 \phi} \sqrt{\frac{t}{\pi}}\right.} \\
& \left.-\frac{(\phi-1)}{2 \phi^{2}} t+\cdots\right]
\end{aligned}
$$

Unfortunately, Equation (61) converges slowly except for very small $t$.

\section{Parametric Approximation}

Practical values of the parameter $\phi$ were found to exceed 400. For $\phi>400$, $\sqrt{s}$ is never more than $2.5 \%$ of $(1+$ $\sqrt{s}+\phi s$ ) for all real values of $s$ from zero to infinity. If $\sqrt{s}$ is neglected in $(1+\sqrt{s}+\phi s)$, the inversion is readily performed and gives

$$
T=1-e^{-t / \phi}
$$

Equation (62), which can also be obtained by physical reasoning, is found to differ from Equation (57) by less than $3 \%$ for all $t$ at $\phi=400$. This is an example of a formal procedure which is quite unsound mathematically, but which leads to a useful and verifiable result. Due caution should be exercised in using this formal procedure because a very small error in representing $f(s)$ may lead to a much larger error in $F(t)$.

\section{Long-time Approximation}

For long times and $L^{3} \gg 3 \phi$, as $s \rightarrow 0$

$$
u \rightarrow \frac{3}{L^{3} s^{2}}
$$

and

$$
T \rightarrow \frac{3 t}{L^{3}}
$$

The form of Equation (64) and hence of Equation (63) can also be rationalized physically.

\section{General Approximation}

It is apparent that the expression

$$
u=\frac{1}{s}\left(\frac{1}{1+\phi s}+\frac{3}{L^{3} s}\right)
$$

yields asymptotic solutions for both long and short times provided that $\phi>400$ and $L^{3} \gg 3 \phi$. Direct numerical comparison shows that Equation (65) differs from Equation (55) by no more than $8 \%$, even in the intermediate range of real values of $s$ where both the long-and shorttime terms are appreciable. Inversion of Equation (65) yields

$$
T=1-e^{-t / \phi}+\frac{3 t}{L^{3}}
$$

Again an approximation which is unjustifiable mathematically appears to give a physically acceptable solution.

\section{Problem IV}

Transient conduction in a semiinfinite slab with radiation and convection from the surface and generation of energy due to a chemical reaction at the surface can be used to illustrate the approximation of boundary conditions. The boundaryvalue problem can be written

$$
\begin{gathered}
k \frac{\partial^{2} T}{\partial z^{2}}=\rho c \frac{\partial T}{\partial t} \\
h\left[T_{s}-T\right]+\sigma\left(T_{s}^{4}-T^{4}\right)+A e^{-B / T^{T}} \\
+k \frac{\partial T}{\partial z}=0 \text { at } z=0
\end{gathered}
$$

and

$$
T=0 \text { at } \quad t=0
$$

Approximate Representation of Radiation

The awkward radiation term can be replaced by a linear expression

$$
h_{r}\left[T_{s}-T^{\prime}\right]=\sigma\left[T_{s}^{4}-T^{4}\right]
$$

where

$$
h_{r}=\sigma\left[T_{s}+T\right]\left[T_{s}{ }^{2}+T^{2}\right]
$$

For any limited range of $T$ the variation of $h_{r}$ will be slight and a mean value can be selected which leads to only a slight error.

Approximate Representation of Generation

Similarly, over a moderate range of temperature the exponential expression can be replaced by a linear expression

$$
A e^{-B / T} \cong a+b T
$$

with the cocfficients $a$ and $b$ chosen empirically.

\section{Solution}

After introduction of the approximate boundary conditions the problem can be rewritten in the following simplified form:

$$
\frac{\partial T}{\partial t}=\frac{\partial^{2} T}{\partial Z^{2}}
$$

$$
\begin{gathered}
T=0 \text { at } \theta=0 \\
T_{0}-T+\frac{\partial T}{\partial Z}=0 \text { at } Z=0
\end{gathered}
$$

where

$$
\begin{gathered}
Z=\left(h+h_{r}-b\right) z / k \\
\sqrt{\theta}=\left(h+h_{r}-b\right) \sqrt{t / k \rho c}
\end{gathered}
$$

and

$T_{0}=\left[a+\left(h_{r}+h\right) T_{s}\right] /\left(h+h_{r}-b\right)$

The rewritten problem can be transformed and the transformed problem solved to give

$$
u=\frac{T_{0} e^{-\sqrt{s} z}}{s(\sqrt{s}+1)}
$$

Equation (79) is then inverted to give

$$
\begin{aligned}
\frac{T}{T_{0}}=\operatorname{erfc}\left(\frac{Z}{2 \sqrt{\theta}}\right)-e^{z+\theta} \\
\operatorname{erfc}\left(\frac{Z}{2 \sqrt{\theta}}+\sqrt{\theta}\right)
\end{aligned}
$$

\section{MACHINE INVERSION}

A general card program has been developed (13) for the inversion of the Laplace transform of functions which can be expressed as a rational algebraic function $a(s) / b(s)$ with a numerator of any order up to fifteen and a denominator of any order up to sixteen. A further restriction is that the poles of the function be of the following types: first-order poles, real or complex; second-order poles, real only; and first-, second-, or thirdorder poles at the origin. The process described requires that the poles be known in advance. However, the roots of such equations can also be found to any desired degree of accuracy by routine machine computation, and so the entire operation can be programmed. 
Obviously, any function which can be approximated by a function of this form can be inverted on a computing machine. A program to evaluate the constants in such an empirical equation can also be written. Thus the entire process of empirical representation, evaluation of singularities, and inversion can be carried out formally by machine. Since the use of the empirical representation is not rigorous, the solution would have to be tested.

\section{CONCLUSIONS}

Complex Laplace transforms can be inverted approximately by a number of simple techniques. Although the validity of the techniques is not easy to establish rigorously, the results can usually be tested by physical reasoning. By the use of empirical representations, almost any function can be inverted on a computing machine. These techniques extend the usefulness of operational calculus to many complex problems in chemical engineering.

\section{ACKNOWLEDGMENT}

Invaluable advice and assistance were provided by J. H. Chin.

\section{NOTATION}

$$
\begin{aligned}
c & =\text { heat capacity } \\
C_{0}= & \text { original concentration of com- } \\
& \text { ponent } 1 \\
C_{n}(t)= & \text { concentration of } n \text {th compo- } \\
& \text { nent }
\end{aligned}
$$

$c(s)=$ transformed concentration of $n$th component

$\operatorname{erfc}(x)=$ complementary error function of $x$

$h=$ heat transfer coefficient for convection

$h_{r} \quad=$ heat transfer coefficient for radiation

$I_{0}(x)=J_{0}(i x)$

$J_{0}(x)=$ Bessel function of first kind and zero order of $x$

$J_{1}(x)=$ Bessel function of first kind and first order of $x$

$K_{1 / 4}(x)=$ modified Bessel function of second kind and one-quarter order of $x$

$k \quad=$ thermal conductivity

$k_{n} \quad=$ rate constant for $n$th reaction

$L=$ dimensionless distance between spheres

$=$ fraction of radius

$=$ variable of transformation

$t \quad=$ time

$T=$ temperature or dimensionless temperature

$T_{s} \quad=$ temperature of surroundings

$u(s)=$ transformed temperature or dimensionless temperature

$z \quad=$ distance from surface

$\phi \quad=$ dimensionless parameter

$\rho=$ density

\section{LITERATURE CITED}

1. Carslaw, H. S., and J. C. Jaeger, "Conduction of Heat in Solids," Oxford
University Press, London (1947).

2. Churchill, R. V., "Modern Operational Mathematies in Engineering," MeGrawHill Book Company, Inc., New York (1944).

3. Doetsch, G., $J$, reine u. angew. Math., 167, 274 (1932).

4. Erdélyi, Arthur, "Tables of Integral Transforms," vol. I, MeGraw-Hill Book Company, Inc., New York (1954).

5. Jeffreys, H., "Operational Methods in Mathematical Physies," Cambridge University Press, London (1931).

6. Marshall, W. R., Jr., and R. L. Pigford, "The Application of Differential Equations to Chemical Engineering Problems," Univ. of Delaware, Newark, Del. (1947).

7. MaLachlan, N. W., "Complex Variable Theory and Transform Calculus," Cambridge University Press, London (1953).

8. Rose, Arthur, J. A. Schilk, and R. C. Johnson, Ind. Eng. Chem., 45, 933 (1953).

9. Rose, Arthur, R. L. Heiny, R. C. Johnson, and J. A. Schilk, ibid., 46, 916 (1954).

10. Rose, Arthur, R. C. Johnson, and R. L. Heiny, ibid., 47, 626 (1955).

11. Rose, Arthur, R. C. Johnson, R. L. Heiny, and T. J. Williams, ibid., 48, $622(1956)$

12. Sleicher, C. A., Jr., and S. W. Churchill, Ind. Eng. Chem., 48, 1819 (1956).

13. Titus, C. K., J. Assoc. Computing Machines, 2, 18 (1955).

14. Widder, D. V., "The Laplace Transform," Princeton University Press, Princeton, N. J. (1946).

\section{Fluidization and Sedimentation of Spherical Particles}

THOMAS J. HANRATTY and ABDEMANNAN BANDUKWALA

Data are presented in support of an expression describing the relation between the sedimenting velocity or the fluidizing velocity and the fraction voids. This expression which contains no empirical constants may be obtained by considering a particle in a fluid having the average properties of the suspension. Stokes's law is used to calculate the force on the particle, and an equation derived by Vand is used to describe the viscosity of the suspension. The equation based on this model is valid for particle Reynolds numbers less than 0.07. The model may be used as an approximation of bed behavior at higher Reynolds numbers by application of a correction to Stokes's law.

The steady state settling rate of a single particle in a fluid or the fluid velocity necessary to suspend a single particle may be described by equating the force of gravity to the viscous drag of the fluid. For spherical particles

Abdemannan Bandukwala is with Standard Oi Company (Indiana), Whiting, Indiana.
Drag force on particle

$$
=C_{D} \frac{1}{2} \rho U_{0}^{2}\left(\frac{\pi D_{p}^{2}}{4}\right)
$$

Force of gravity

$$
=\left(\rho_{s}-\rho\right) g\left(\frac{\pi D_{p}{ }^{3}}{6}\right)
$$

University of Illinois, Urbana, Illinois

$$
U_{0}=\sqrt{\frac{4 g D_{\nu}\left(\rho_{s}-\rho\right)}{3 \rho C_{D}}}
$$

For low settling rates $\left(R e_{p}<0.1\right)$ the drag coefficient $C_{D}$ may be described by Stokes's law, and Equation (3) becomes

$$
U_{0}=\frac{\left(\rho_{s}-\rho\right) g D_{p}^{2}}{18 \mu}
$$

